\title{
Approximations of the inverse wavelet transform for analogue circuits
}

\author{
Marco A. Gurrola-Navarro ${ }^{1 a)}$, Agustín S. Medina-Vazquez ${ }^{1}$, \\ and Guillermo Espinosa-Flores-Verdad ${ }^{2}$ \\ ${ }^{1}$ Departamento de Electrónica, CUCEI, Universidad de Guadalajara \\ Av. Revolución 1500, Guadalajara, Jalisco, México, CP 44430 \\ ${ }^{2}$ Instituto Nacional de Astrofísica Optica y Electrónica \\ Luis Enrique Erro \# 1, Tonantzintla, Puebla, México, CP 72840 \\ a)marco.gurrola@cucei.udg.mx
}

\begin{abstract}
We propose two approximations of the inverse wavelet transform implemented with a voltage adder and two analog filters. They work together with a set of scaled band-pass analog filters that perform the wavelet transform of a continuous time signal. With this approach an integrated circuit has been fabricated. On-chip measurements demonstrate signal to reconstruction error ratios up to $25.8 \mathrm{~dB}$.
\end{abstract}

Keywords: wavelet transform, integrated circuits, analog circuits

Classification: Electron devices, circuits, and systems

\section{References}

[1] S. A. P. Haddad and W. A. Serdijn, Ultra Low-Power Biomedical Signal Processing: An analog wavelet filter approach for pacemakers, Springer, Dordrecht, 2009.

[2] M. A. Gurrola-Navarro and G. Espinosa-Flores-Verdad, "Analogue wavelet transform with single biquad stage per octave," IET Electronincs Letters, vol. 46, no. 9, pp. 616-618, April 2010.

[3] S. Mallat and S. Zhong, "Characterization of signals from multiscale edges," IEEE Trans. Pattern Anal. Mach. Intell., vol. 14, no. 7, pp. 710732 , July 1992.

[4] M. Bikumandla, J. Ramírez-Angulo, C. Urquidi, R. G. Carvajal, and A. J. Lopez-Martin, "Biasing CMOS amplifiers using MOS transistors in subthreshold region," IEICE Electron. Express, vol. 1, no. 12, pp. 339345, Sept. 2004.

\section{Introduction}

The Wavelet Transform (WT) is commonly used as a tool to perform a simultaneous time-frequency analysis of transitory features in time signals. Some processing techniques that are applied when the signal is in the wavelet domain require the application of the Inverse Wavelet Transform (IWT) in order to take the processed signal back to time domain. 
Even when most of the reported implementations of the WT and IWT have been numerically performed, there has also been interest in finding analog implementations. For example, in [1] a methodology is presented to design analog filters (intended for low power bioimplantable devices) whose Impulse Response (IR) is an approximation of a given mother wavelet. Moreover, in [2] it is shown that the IR of a biquadratic band-pass filter fulfils the admissibility and stability constraints to be considered as an exact wavelet. Thus, a complete WT system can be created with a set of biquad scaled filters, where the set of output signals is the wavelet domain representation of the input signal.

Now this work discusses the problem of recovering the original signal from the wavelet domain using an analog implementation of the IWT.

\section{Ideal wavelet transform and inverse wavelet transform sys- tem}

Fig. $1 a$ shows an ideal system performing the WT and the IWT of a continuous time signal, expressed in the frequency domain. It contains an adder and two sets of scaled filters, $H\left(r^{m} \omega\right)$ and $\mathrm{X}\left(r^{m} \omega\right)$, where $r>1$ is the constant of scaling between adjacent filters, $m=\ldots,-1,0,1,2, \ldots, \omega=2 \pi f$, and $f$ is frequency in Hz. A necessary condition to perform the WT is that $H(\omega)$ must be a band-pass filter. $F(\omega)$ and $F_{\text {out }}(\omega)$ are the Fourier transforms of the continuous input signal, $f(t)$, and the output signal, $f_{\text {out }}(t)$, respectively. We will denote the IR of $H\left(r^{m} \omega\right)$ as $h_{r^{m}}(t)$, then we can obtain that

$$
h_{r^{m}}(t)=\left(1 / r^{m}\right) h_{r^{0}}\left(t / r^{m}\right) .
$$

The output of $H\left(r^{m} \omega\right)$, given by $F(\omega) H\left(r^{m} \omega\right)$, will be denoted in time domain as $W_{r^{m}} f(t)$. Then, $W_{r^{m}} f(b)=\int_{-\infty}^{\infty} f(t) h_{r^{m}}(b-t) d t$. This is the formal definition of the dyadic WT given in [3] (for the case $r=2$ ), where $W_{r^{m}} f(b)$ is the WT at translation time $b$, with respect to the mother wavelet, $h_{r^{0}}(-t)$, scaled to the $m$-th scale. In this work we use the more general term of semidiscrete WT when the transform is continuous in translation and discrete in scale. This covers any value $r>1$. In the system of Fig. 1a, the IWT is performed by the set of filters $\mathrm{X}\left(r^{m} \omega\right)$ and an adder.

Note that a given system will produce an output signal equal to its input signal only if its Transfer function (TF) is equal to 1, i.e., the complex TF must have a magnitude of 1 and a phase angle of 0 over the system frequency range. In the case of the ideal WT-IWT system, this condition for perfect reconstruction is $\sum_{m=-\infty}^{\infty} H\left(r^{m} \omega\right) \mathrm{X}\left(r^{m} \omega\right)=1$, for $\omega \in(0, \infty)[3]$.

\section{Approximations of the semidiscrete inverse wavelet trans- form}

We present two approximations of the ideal WT-IWT system. The simple approximation is the system of Fig. $1 b$ working with the switches opened. For circuital simplification, we have taken only a finite number of filters, $(M+1)$, and given a constant value to all filters $\mathrm{X}\left(r^{m} \omega\right)=\chi$. This new system 


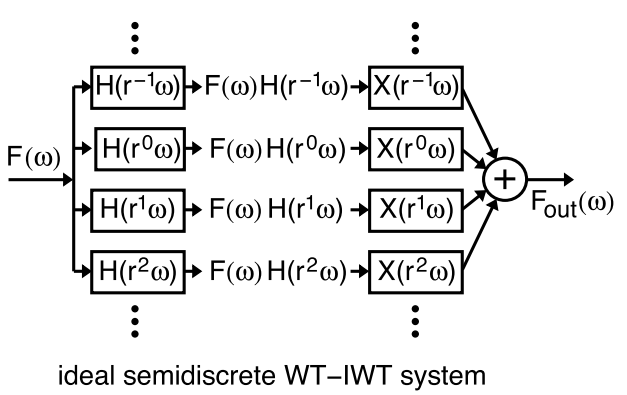

(a)

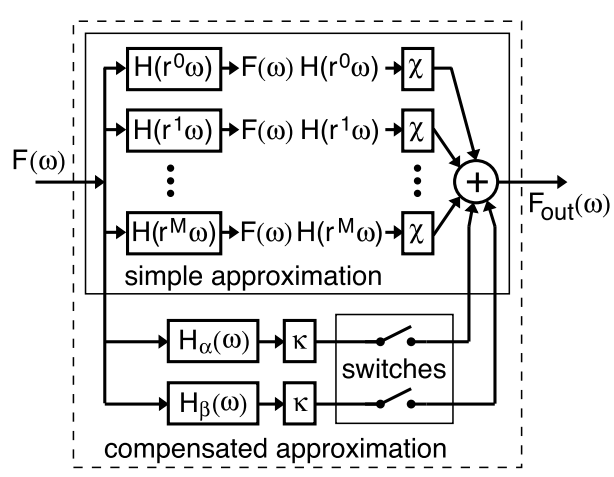

(b)

Fig. 1. Frequency domain descriptions of semidiscrete WT-IWT systems (a) Ideal system (b) Proposed approximations

works only along a finite range of frequency covered by the band-pass filters $H\left(r^{m} \omega\right)$. We will denote this frequency range as $L$. For this approximation, the condition for perfect reconstruction is $\sum_{m=0}^{M} \chi H\left(r^{m} \omega\right)=1$, for $\omega \in L$.

To validate the proposed approach, the simple approximation has been on-chip fabricated for $H(\omega), r, M$, and $\chi$ given in Table I. In [2] it is shown that the IR of this $H(\omega)$ satisfies the mathematical conditions to be used as a mother wavelet. The $\chi$ value is selected to normalize the system gain. As can be seen in Fig. $2 a$, the gain is within $1.000 \pm 0.005$ (very close to 1 ) from $100 \mathrm{~Hz}$ to $10 \mathrm{kHz}$. We will take this frequency range as $L$. For its part, the phase response on $L$ is within $0^{\circ} \pm 30.2^{\circ}$, far from the required zero phase shift (see Fig. 2b).

Table I. Selected values for on-chip implementation of system in Fig. $1 b$, for a range $L=[100 \mathrm{~Hz}, 10 \mathrm{kHz}]$

\begin{tabular}{|l|l|l|}
\hline$H\left(r^{m} \omega\right)=\frac{5.978 \times 10^{4}\left(j r^{m} \omega\right)}{\left(j r^{m} \omega\right)^{2}+5.978 \times 10^{4}\left(j r^{m} \omega\right)+7.146 \times 10^{9}}$ & $r=\sqrt{2}$ & $M=15$ \\
\hline$H_{\alpha}(\omega)=\frac{(j \omega)^{2}+1.504 \times 10^{5}(j \omega)}{(j \omega)^{2}+1.504 \times 10^{5}(j \omega)+1.584 \times 10^{10}}$ & $\chi=0.3794$ & $\kappa=0.78$ \\
\hline$H_{\beta}(\omega)=\frac{375.0(j \omega)+9.842 \times 10^{4}}{(j \omega)^{2}+375.0(j \omega)+9.842 \times 10^{4}}$ & $j=\sqrt{-1}$ & $N=20$ \\
\hline
\end{tabular}

However, for the values in Table I we have numerically observed that $\sum_{m=-N}^{M+N} \chi H\left(r^{m} \omega\right)$ holds a magnitude near 1 , and flattens the phase close to $0^{\circ}$, on $L$, as the non-negative integer $N$ grows, as shown in Fig. $2 a$ and $2 b$ for $N=0,2,4$. Now, if we define $\kappa H_{\alpha}(\omega) \approx \sum_{m=-N}^{-1} \chi H\left(r^{m} \omega\right)$ and $\kappa H_{\beta}(\omega) \approx$ $\sum_{m=M+1}^{M+N} \chi H\left(r^{m} \omega\right)$ over the range $L$, for a real $\kappa$, and a sufficiently large $N$, it is possible to corroborate that $\sum_{m=0}^{M} \chi H\left(r^{m} \omega\right)+\kappa H_{\alpha}(\omega)+\kappa H_{\beta}(\omega) \approx$ $\sum_{m=-N}^{M+N} \chi H\left(r^{m} \omega\right) \approx 1$, for $\omega \in L$ with almost flat phase (see Fig. $2 b$ ). In this manner, the implemented compensated approximation (Fig. $1 b$ with switches closed) overcomes the phase problem on $L$.

For the manufactured chip, the $H_{\alpha}(\omega), H_{\beta}(\omega), \kappa$, and $N$ values are given 


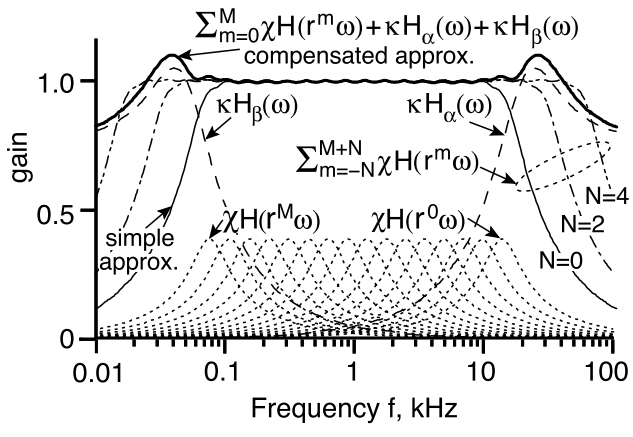

(a)

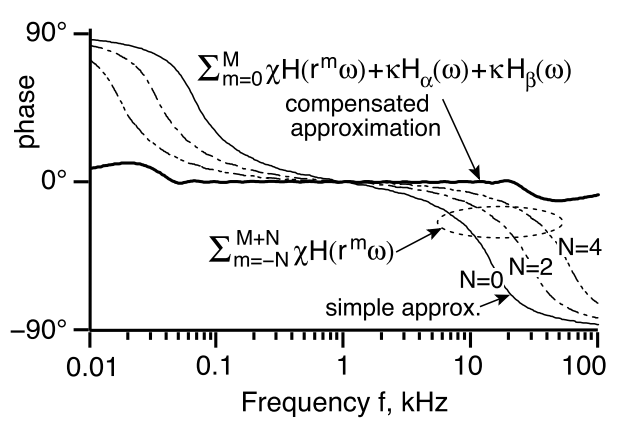

(b)

Fig. 2. Frequency response of approximations in Fig. $1 b$ for the values in Table I (numerical simulations) (a) Magnitude (b) Phase angle

in Table I. This compensated system has a gain of $1.000 \pm 0.007$, and a phase angle of $0.0^{\circ} \pm 0.4^{\circ}$, over the range $L$ (see Fig. $2 a$ and $2 b$ ).

Note that we could select different $H(\omega), r, M$ and $\chi$ values than those given in Table I, but a good reconstruction can be done only if the TF of the system has an approximate gain of 1 and phase of $0^{\circ}$ over the system frequency range, $L$. Moreover, we could select filters $H_{\alpha}(\omega)$ and $H_{\beta}(\omega)$ with different shapes than those given in Table I, but their coefficients have to be numerically fitted to approximate the above indicated partial sums on $L$, for a sufficiently large $N$ to flat the phase in this frequency range.

\section{Circuit implementation}

The approximations of Fig. $1 b$ (for the Table I values) have been fabricated in a $0.5 \mu \mathrm{m} \mathrm{N}$-well CMOS process. Filters $H\left(r^{0} \omega\right)$ to $H\left(r^{15} \omega\right)$ are implemented with the band-pass filters $F_{0}$ to $F_{15}$ of Fig. $3 a$, with TF $\frac{v_{k}(\omega)}{v_{i n}(\omega)}=$ $\frac{C_{a, k} G_{k}(j \omega)}{C_{a, k}\left(C_{b, k}+C_{c, k}\right)(j \omega)^{2}+C_{a, k} G_{k}(j \omega)+G_{k}^{2}}$, for $j=\sqrt{-1}, k=0, \ldots, 15$, capacitor values given in Fig. $3 a$ and transconductance, $G_{k}=G_{a, k}=G_{b, k}$, set as follows. The central frequency of the band-pass filters, $F_{k}$, has to be exponentially scaled by a factor $r^{k}$, as required by $H\left(r^{k} \omega\right)$ given in Table I. It is achieved by scaling exponentially the transconductance $G_{k}$ of the Operational Transconductance Amplifier (OTA) of Fig. 3b. This OTA has a differential input range of $\pm 29 \mathrm{mV}$ within $1 \%$ of linearity [2]. The OTA transistors have the following dimensions in $\mu \mathrm{m} / \mu \mathrm{m}: 36 / 4.5$ for $M_{1}$ to $M_{4}, 17 / 4.5$ for $M_{5}$ and $M_{6}$, and 10/4.5 for $M_{7}$ and $M_{8}$. Transistors are biased in subthreshold region, where the current $I_{b}$ (and the OTA transconductance $G$ that is proportional to $I_{b}$ ) has exponential dependence with respect to nearly linear changes in the bias voltage $V_{b}$ [2]. The bias voltages $V_{b 0}$ to $V_{b 15}$ are produced with the help of a ladder of 15 poly resistors of nearly equal value, $R_{1}$ to $R_{15}$ [2].

Filters $H_{\alpha}(\omega)$ and $H_{\beta}(\omega)$ were implemented with the Gm-C filters $F_{\alpha}$, and $F_{\beta}$ shown in Fig. $3 a$, with TF $\frac{v_{\alpha}(\omega)}{v_{i n}(\omega)}=\frac{C_{d} C_{e}(j \omega)^{2}+C_{e} G_{\alpha}(j \omega)}{C_{d} C_{e}(j \omega)^{2}+C_{e} G_{\alpha}(j \omega)+G_{\alpha}^{2}}$, and $\frac{v_{\beta}(\omega)}{v_{i n}(\omega)}=$ $\frac{C_{g} G_{\beta}(j \omega)+G_{\beta}^{2}}{C_{g}\left(C_{h}+C_{\beta}\right)(j \omega)^{2}+C_{g} G_{\beta}(j \omega)+G_{\beta}^{2}}$, respectively, where $G_{\alpha}=G_{a \alpha}=G_{b \alpha}$, and $G_{\beta}=$ $G_{a \beta}=G_{b \beta}$. 


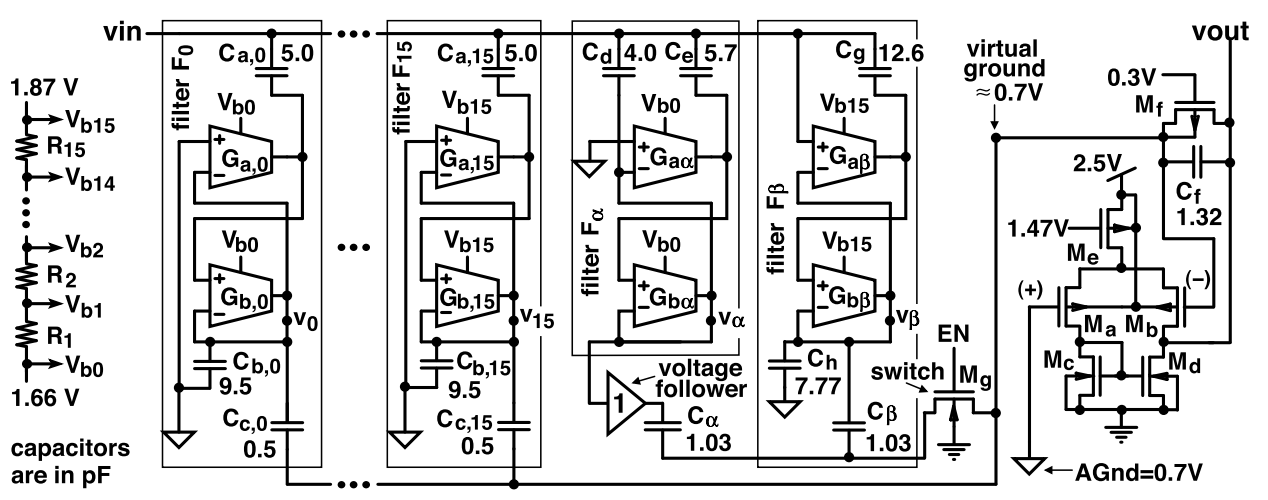

(a)

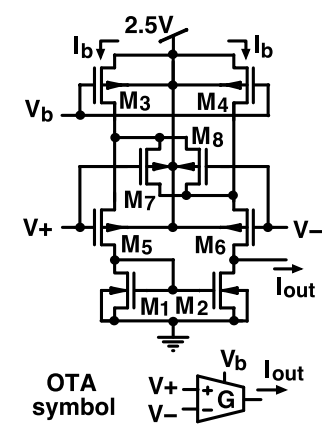

(b)

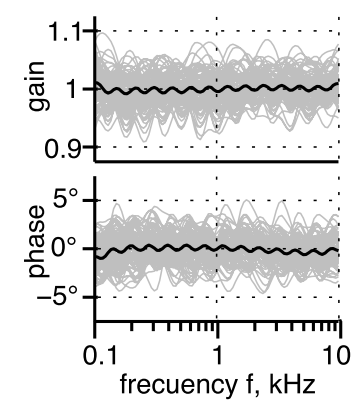

(c)

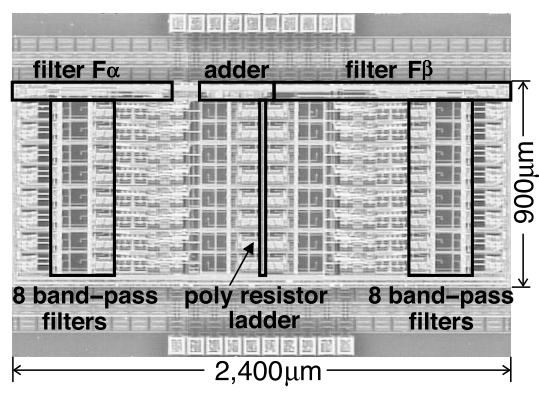

(d)

Fig. 3. Integrated circuit implementation (a) Schematic circuit (b) Operational Transconductance Amplifier (OTA) (c) Typical (black) and MC (gray) simulations of circuit in Fig. $3 a$ with $E N$ active (d) Microphotograph of system in manufactured chip

A five-transistor operational amplifier $\left(M_{a}\right.$ to $M_{e}$ in Fig. $\left.3 a\right)$ is used in inverter adder configuration, where $C_{c, k}, C_{\alpha}$, and $C_{\beta}$ are the input impedances, and $C_{f}$ is the feedback impedance [4]. Transistors $M_{a}$ to $M_{e}$, with aspect ratio $100 \mu \mathrm{m} / 0.9 \mu \mathrm{m}$, work in the saturation region. Transistor $M_{f}$ $(0.9 \mu \mathrm{m} / 0.6 \mu \mathrm{m})$ is used in subthreshold region as a high-value resistor to hold the DC voltage level of $C_{f}$ near zero [4]. The voltage follower is implemented with a MOSFET-P in source follower configuration. The pass transistor $M_{g}$ is controlled by the logic signal $E N$. The adder operation is given by $v_{\text {out }}(t)=\sum_{k=0}^{15}\left(-C_{c, k} / C_{f}\right) v_{k}(t)$ when $E N=0$, and by $v_{\text {out }}(t)=$ $\sum_{k=0}^{15}\left(-C_{c, k} / C_{f}\right) v_{k}(t)-\left(C_{\alpha} / C_{f}\right) v_{\alpha}(t)-\left(C_{\beta} / C_{f}\right) v_{\beta}(t)$ when $E N=1$.

To explore the effect of the process variations on the circuit response, Fig. $3 c$ shows 100 Monte Carlo (MC) AC transistor level simulations of node $v_{\text {out }}$ with respect to $v_{\text {in }}$ for the compensated approximation case. $68 \%$ of the MC simulations had a magnitude within $1.000 \pm 0.065$ and a phase angle of $0.0^{\circ} \pm 4.6^{\circ}$ on $L$. The typical simulation is also shown in Fig. $3 c$. It is very close to the compensated approximation of Fig. $2 a$ and $2 b$.

\section{Experimental results}

Fig. $3 d$ shows a microphotograph of the manufactured chip. The system blocks cover an area of $0.66 \mathrm{~mm}^{2}$ and have a power consumption about $14 \mu \mathrm{W}$. 
A probe signal made up of 4 sinusoidal cycles of period $T, \pm 10 \mathrm{mV}$ of amplitude and a sinusoidal envelope of $8 T$ period has been prepared. It was periodically applied to node $v_{i n}$. Averages over 512 measures of $v_{k}(t)$ and $v_{\text {out }}(t)$ were taken to reduce the environmental noise effect. Fig. $4 a$ shows the probe signal for $T=4 \mathrm{~ms}$, and its WT plotted with the absolute value of the measured voltages $v_{0}(t)$ to $v_{15}(t)$. Fig. $4 b$ shows the measured reconstructed signal $v_{\text {out }}(t)$ for both simple and compensated approximations, for $T=4 \mathrm{~ms}$.

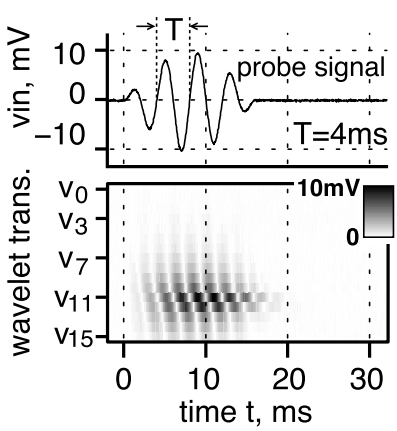

(a)

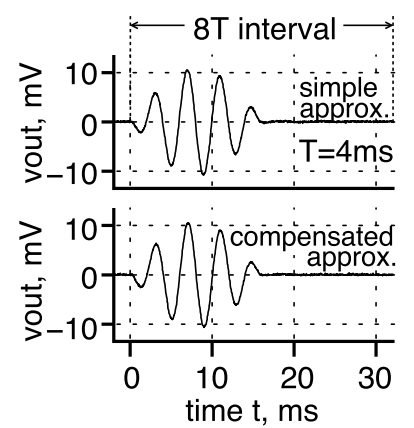

(b)

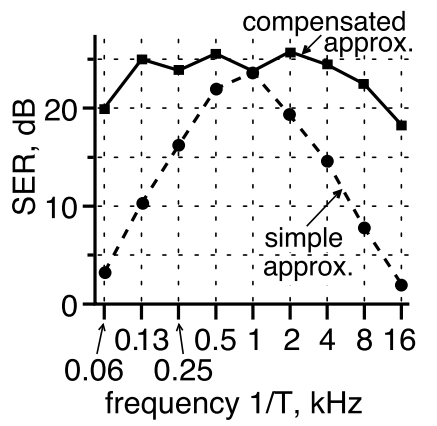

(c)

Fig. 4. On chip tests (a) Probe signal for $T=4 \mathrm{~ms}$ and its wavelet transform components $v_{0}$ to $v_{15}$ (b) Reconstructions of probe signal for $T=4 \mathrm{~ms}$ (c) SER for various $1 / T$ frequencies

It can be verified, that most of the probe signal energy spectrum is around frequency $1 / T$. In both approximations, the probe signal was applied for, $T=\left\{2^{-4}, 2^{-3}, \ldots, 2^{4}\right\} \mathrm{ms}$. We have to mention that the system gain was intentionally set to a value slightly greater than 1 . Then we take amplification, $A$, as the ratio between the peak-to-peak voltages of the output and input signals. The measured average amplification for all the $T$ values was $\bar{A}=-1.08$. Now, we define the Signal to (normalized) reconstruction Error Ratio as SER $=10 \log _{10}\left(\int_{0}^{8 T}\left|v_{\text {in }}(t)\right|^{2} d t / \int_{0}^{8 T}\left|v_{\text {out }}(t) / \bar{A}-v_{\text {in }}(t)\right|^{2} d t\right)(\mathrm{dB})$. The interval of integration used, $8 T$, is explained in Fig. $4 b$. Fig. $4 c$ plots the SER vs. $1 / T$. Both approximations have a similar maximum SER that is limited due to the effect of the process variations. The compensated approximation holds the SER near $25 \mathrm{~dB}$ all along $L$. However, the simple approximation has its maximum only at $1 \mathrm{kHz}$, where the numerical model presents a zero phase angle shift (see Fig. $2 b$ ). Below and above $1 \mathrm{kHz}$, the increase in the phase absolute value produces a decrease in the SER.

\section{Conclusion}

An approach for implementing the inverse wavelet transform using simple analog circuits has been presented. The theoretical concept has been validated by means of a fabricated chip. Signal to reconstruction error ratios up to $25 \mathrm{~dB}$ have been measured. 